\title{
SMALL ZEROS OF QUADRATIC FORMS OVER NUMBER FIELDS. II
}

\author{
JEFFREY D. VAALER
}

\begin{abstract}
Let $F$ be a nontrivial quadratic form in $N$ variables with coefficients in a number field $k$ and let $\mathscr{Z}$ be a subspace of $k^{N}$ of dimension $M, 1 \leq M \leq N$. If $F$ restricted to $\mathscr{Z}$ vanishes on a subspace of dimension $L, 1 \leq L<M$, and if the rank of $F$ restricted to $\mathscr{Z}$ is greater than $M-L$, then we show that $F$ must vanish on $M-L+1$ distinct subspaces $\mathscr{Z}_{0}, \mathscr{Z}_{1}, \ldots, \mathscr{X}_{M-L}$ in $\mathscr{Z}$ each of which has dimension $L$. Moreover, we show that for each pair $\mathscr{Z}_{0}, \mathscr{L}_{1}, 1 \leq l \leq M-L$, the product of their heights $H\left(\mathscr{Z}_{0}\right) H\left(\mathscr{Z}_{1}\right)$ is relatively small. Our results generalize recent work of Schlickewei and Schmidt.
\end{abstract}

\section{INTRODUCTION}

Let

$$
F(\mathbf{x}, \mathbf{y})=\sum_{m=1}^{N} \sum_{n=1}^{N} \varphi_{m n} y_{m} x_{n}
$$

be a symmetric bilinear form with coefficients $\varphi_{m n}=\varphi_{n m}$ in an algebraic number field $k$. We write $\Phi=\left(\varphi_{m n}\right)$ for the associated $N \times N$ matrix and $F(\mathbf{x})=F(\mathbf{x}, \mathbf{x})$ for the associated quadratic form. As in our earlier paper [14] we will consider $F$ restricted to a fixed subspace $\mathscr{Z} \subseteq k^{N}$ of dimension $M$, $1 \leq M \leq N$, and define

$$
\mathscr{Z}^{(0)}=\{\mathbf{z} \in \mathscr{Z}: F(\mathbf{z})=0\} .
$$

A basic problem in this situation is to show that if $\mathscr{Z}^{(0)}$ is not trivial then it necessarily contains vectors or subspaces of small height. Beginning with a result of Cassels [3, 4], the papers [1, 5-10, 12-15], are all directed at this type of problem. In case $k=\mathbb{Q}$ and $M=N$ Schlickewei and Schmidt [10] have recently proved a theorem which includes most of the previous results. Our purpose in the present paper is to generalize the work of Schlickewei and Schmidt to an arbitrary number field $k$ and to the case where $\mathscr{Z}$ may be a proper subspace of $k^{N}$. As we have already noted in [14], the introduction of the subspace $\mathscr{Z}$ is equivalent to considering the simultaneous zeros of the

Received by the editors January $21,1988$.

1980 Mathematics Subject Classification (1985 Revision). Primary 11D09; Secondary 11 H55.

Research supported by a grant from the National Science Foundation, DMS-8701396. 
quadratic form $F$ and a system of $N-M$ independent linear forms. If $A$ is an $(N-M) \times N$ matrix over $k$ with $\operatorname{rank}(A)=N-M$ and

$$
\mathscr{Z}=\left\{\mathbf{x} \in k^{N}: A \mathbf{x}=\mathbf{0}\right\},
$$

then by a basic duality principle (equation (2.6) of [14]) the subspace $\mathscr{Z}$ and the matrix $A$ have the same height. For this reason all of our results which we state for a quadratic form $F$ restricted to $\mathscr{Z}$ have obvious analogs for the set of simultaneous solutions in $k^{N}$ of $F(\mathbf{x})=0$ and $A \mathbf{x}=\mathbf{0}$.

We suppose that the number field $k$ has degree $d$ over $\mathbb{Q}$. Our notation for places, completions of $k$, normalized absolute values, heights and measures will be identical to that which was already described in $[14, \S 2]$. Also, we will assume that $F$ restricted to $\mathscr{Z}$ has rank $r$. Our main result which generalizes Theorem 1 in [10] is as follows.

Theorem 1. Suppose that $\mathscr{Z}^{(0)}$ contains an L-dimensional subspace, $1 \leq L<$ $M$, and $F$ restricted to $\mathscr{Z}$ has rank $r>M-L$. Then there exist $M-L+1$ distinct L-dimensional subspaces $\mathscr{X}_{0}, \mathscr{X}_{1}, \mathscr{X}_{2}, \ldots, \mathscr{X}_{M-L}$ in $\mathscr{Z}^{(0)}$ with the following properties:

(i) for each $l=1,2, \ldots, M-L$ the subspace $\mathscr{X}_{0} \cap \mathscr{X}_{l}$ has dimension $L-1$;

(ii) the union $\mathscr{X}_{0} \cup \mathscr{X}_{1} \cup \cdots \cup \mathscr{X}_{M-L}$ spans the subspace $\mathscr{Z}$;

(iii) for each $l=1,2, \ldots, M-L$,

$$
H\left(\mathscr{X}_{0}\right)^{2} \leq H\left(\mathscr{X}_{0}\right) H\left(\mathscr{X}_{l}\right) \leq\left\{2 c_{k}(M-L)^{2} \mathscr{H}(\Phi)\right\}^{M-L}\left\{2 c_{k}(1) H(\mathscr{Z})\right\}^{2} .
$$

(For each positive integer $n, c_{k}(n)$ is a field constant defined in [14, §2] and in (2.2) below.)

In our previous result [14, Theorem 1] we made no assumption concerning the rank of $F$ restricted to $\mathscr{Z}$ but instead we assumed that the integer $L$ was maximal. That is, we assumed that $L$ was the largest integer such that $\mathscr{Z}^{(0)}$ contains a subspace of dimension $L$. We note that in the present paper we are not assuming that $L$ is maximal. Of course $\mathscr{Z}^{(0)}$ must contain the subspace

$$
\mathscr{Z}^{\perp}=\{\mathbf{z} \in \mathscr{Z}: F(\boldsymbol{\zeta}, \mathbf{z})=0 \text { for all } \zeta \in \mathscr{Z}\},
$$

and $\operatorname{dim}\left(\mathscr{Z}^{\perp}\right)=M-r$. Thus our hypothesis in Theorem 1 concerning the rank of $F$ restricted to $\mathscr{Z}$ could be stated this way: we assume that $\mathscr{Z}^{(0)}$ contains a subspace of dimension $L$ with $L>\operatorname{dim}\left(\mathscr{Z}^{\perp}\right)$. It turns out that the method used to prove Theorem 1 also provides a bound on the height of the subspace $\mathscr{Z}^{\perp}$.

Theorem 2. If $1 \leq r<M$ then

$$
H\left(\mathscr{Z}^{\perp}\right) \leq c_{k}(r)^{r} \mathscr{H}(\Phi)^{r / 2} H(\mathscr{Z}) .
$$

Suppose that in Theorem 1 the quadratic form $F$ restricted to $\mathscr{Z}$ has rank $r=M$. Then we may take $L=1$. We find that if $F$ has a nontrivial zero 
in $\mathscr{Z}$, then there is a basis $\left\{\mathbf{x}_{0}, \mathbf{x}_{1}, \ldots, \mathbf{x}_{M-1}\right\}$ of $\mathscr{Z}$ such that $F\left(\mathbf{x}_{m}\right)=0$, $m=0,1, \ldots, M-1$, and

$$
H\left(\mathbf{x}_{0}\right) H\left(\mathbf{x}_{l}\right) \leq\left\{2 c_{k}(M-1)^{2} \mathscr{C}(\Phi)\right\}^{M-1}\left\{2 c_{k}(1) H(\mathscr{Z})\right\}^{2},
$$

for $l=1,2, \ldots, M-1$. Obviously (1.4) implies that

$$
\begin{aligned}
& H\left(\mathbf{x}_{0}\right)^{M-1} H\left(\mathbf{x}_{1}\right) H\left(\mathbf{x}_{2}\right) \cdots H\left(\mathbf{x}_{M-1}\right) \\
& \quad \leq\left\{2 c_{k}(M-1)^{2} \mathscr{H}(\Phi)\right\}^{(M-1)^{2}}\left\{2 c_{k}(1) H(\mathscr{Z})\right\}^{2(M-1)} .
\end{aligned}
$$

The bounds (1.4) and (1.5) generalize results of Schulze-Pillot [13, Theorem 2] and Chalk [5].

If $F$ and $\mathscr{Z}$ satisfy the hypotheses in Theorem 1 then $\mathscr{Z}^{(0)}$ contains the $L$-dimensional subspace $\mathscr{X}_{0}$, and the height $H\left(\mathscr{X}_{0}\right)$ is bounded by (1.2). In fact the slightly sharper bound

$$
H\left(\mathscr{X}_{0}\right) \leq\left\{2 c_{k}(M-L)^{2} \mathscr{H}(\Phi)\right\}^{(M-L) / 2} H(\mathscr{Z})
$$

follows immediately from Theorem 4 and equation (5.11) below. Theorem 2 and (1.6) provide a sharper and more general formulation of our previous result [14, Theorem 1].

We wish to thank Professor H. P. Schlickewei for helpful discussions on the subject of this paper.

\section{Preliminary lemmas}

If $v$ is a place of $k$ we write $k_{v}$ for the completion of $k$ at $v$. Let $\mathscr{A} \subseteq(k)^{N}$ be a subspace of dimension $L, 1 \leq L<N$, and let $\mathscr{A}_{v} \subseteq\left(k_{v}\right)^{N}$ be the completion of $\mathscr{A}$ in $\left(k_{v}\right)^{N}$. We shall make frequent use of the $N \times N$ projection matrices $P_{v}=P_{v}\left(\mathscr{A}_{v}\right)$ which were defined in [14,§4]. Here we simply summarize the main results concerning $P_{v}\left(\mathscr{A}_{v}\right)$ which we will need. Complete proofs and further details can be found in [14].

At each place $v$ the matrix $P_{v}$ is a projection operator in the usual sense:

$$
P_{v} \mathbf{x} \in \mathscr{A}_{v} \text { for all } \mathrm{x} \in\left(k_{v}\right)^{N},
$$

and

$$
P_{v} \mathbf{x}=\mathbf{x} \text { for all } \mathbf{x} \in \mathscr{A}_{v} .
$$

Let $\mathbf{b} \in(k)^{N} \backslash \mathscr{A}$ so that

$$
\mathscr{B}=\operatorname{span}_{k}\{\mathscr{A}, \mathbf{b}\}
$$

is a subspace of dimension $L+1$. The height of the subspaces $\mathscr{A}$ and $\mathscr{B}$ are related by the identity

$$
H(\mathscr{B})=H(\mathscr{A}) \prod_{v} H_{v}\left\{\left(1_{N}-P_{v}\right) \mathbf{b}\right\} .
$$

This follows from [14, Lemma 4]. 
If $\mathscr{A} \subseteq k^{N}$ we shall sometimes simplify our notation and write $P_{v}(\mathscr{A})$ for projection onto the completion of $\mathscr{A}$ in $\left(k_{v}\right)^{N}$.

Next we require a lemma whose proof uses methods from geometry of numbers over adèle spaces. The relevant definitions concerning this subject are contained in [2, pp. 16-18]. We write $k_{\mathrm{A}}$ for the adele ring of the number field $k$ and if $v$ is a finite place of $k$ then

$$
O_{v}=\left\{x \in k_{v}:|x|_{v} \leq 1\right\}
$$

denotes the maximal compact subring of $k_{v}$. Since the additive group of $k_{v}$ is locally compact we may select a normalized Haar measure $\beta_{v}$ on $k_{v}$ as follows:

(i) If $v \mid p$, where $p$ is a (finite) rational prime, we require that $\beta_{v}\left(O_{v}\right)=$ $\left|\mathscr{D}_{v}\right|_{v}^{d / 2}$. Here $\mathscr{D}_{v}$ is the local different of $k$ at $v$.

(ii) If $k_{v}=\mathbf{R}$ then $\boldsymbol{\beta}_{v}$ is ordinary Lebesgue measure on $\mathbf{R}$.

(iii) If $k_{v}=\mathbb{C}$ then $\beta_{v}$ is Lebesgue measure on the complex plane multiplied by 2 .

The product measure $\beta=\prod_{v} \beta_{v}$ then induces a normalized Haar measure (also denoted by $\beta$ ) on $k_{\mathrm{A}}$. If $\left(k_{\mathbf{A}}\right)^{N}$ is an $N$-fold product of adèle spaces we write $V$ for the product Haar measure $\beta^{N}$ on $\left(k_{\mathrm{A}}\right)^{N}$.

At each infinite place $v$ we define a positive real number $r_{v}(N)$ so that

$$
\beta_{v}^{N}\left(\left\{\mathbf{u} \in\left(k_{v}\right)^{N}:\|\mathbf{u}\|_{v}<r_{v}(N)\right\}\right)=1 .
$$

The exact value of $r_{v}(N)$ is given in [14, §2]. We also define

$$
c_{k}(N)=\left\{2\left|\Delta_{k}\right|^{1 / 2 d} \prod_{v \mid \infty}\left(r_{v}(N)\right)^{d_{v} / d}\right\},
$$

where $\Delta_{k}$ is the discriminant of $k$ and $d_{v}=\left[k_{v}: \mathbb{Q}_{v}\right]$ is the local degree. The quantity $c_{k}(N)$ will occur as a field constant.

Now suppose that $A_{v}$ is an $N \times M$ matrix over $k_{v}$ with

$$
\operatorname{rank}\left(A_{v}\right)=M \leq N .
$$

Let $\boldsymbol{\xi}_{v} \neq \mathbf{0}$ be a vector in $\left(k_{v}\right)^{N}$. If $v \mid \infty$ we set

$$
S_{v}=\left\{\mathbf{u} \in\left(k_{v}\right)^{M}:\left\|A_{v} \mathbf{u}\right\|_{v}<\left\|\boldsymbol{\xi}_{v}\right\|_{v}\right\} .
$$

It follows easily that

$$
\beta_{v}^{M}\left(S_{v}\right)=r_{v}(M)^{-M d_{v}}\|\boldsymbol{\xi}\|_{v}^{M d_{v}} H_{v}\left(A_{v}\right)^{-d} .
$$

If $v \nmid \infty$ we write

$$
S_{v}=\left\{\mathbf{u} \in\left(k_{v}\right)^{M}:\left\|A_{v} \mathbf{u}\right\|_{v} \leq\left\|\boldsymbol{\xi}_{v}\right\|_{v}\right\} .
$$

By using the $v$-adic cube slicing identity, which is (4.8) and (4.9) of [2], the Haar measure of $S_{v}$ is given by

$$
\beta_{v}^{M}\left(S_{v}\right)=\left|\mathscr{D}_{v}\right|_{v}^{M d / 2}\left\|\boldsymbol{\xi}_{v}\right\|_{v}^{M d_{v}} H_{v}\left(A_{v}\right)^{-d} .
$$


If we assume that $S_{v}=\left(O_{v}\right)^{M}$ at almost all finite places $v$, then the set

$$
\mathscr{S}=\prod_{v} S_{v}
$$

is a subset of the $M$-fold product $\left(k_{\mathrm{A}}\right)^{M}$. Hence the Haar measure of $\mathscr{S}$ is given by

$$
V(\mathscr{S})=\prod_{v} \beta_{v}^{M}\left(S_{v}\right)=\left|\Delta_{k}\right|^{-M / 2}\left\{\prod_{v}\left|\boldsymbol{\xi}_{v}\right|_{v}^{M} H_{v}\left(A_{v}\right)^{-1}\right\}^{d}\left\{\prod_{v \mid \infty} r_{v}(M)^{-M d_{v}}\right\} .
$$

(In (2.3) we have used the identity

$$
\prod_{v \nmid \infty}\left|\mathscr{D}_{v}\right|_{v}^{d / 2}=\left|\Delta_{k}\right|^{-1 / 2} \text {.) }
$$

If $0<\lambda_{1} \leq \lambda_{2} \leq \cdots \leq \lambda_{M}<\infty$ denote the successive minima of $\mathscr{S}$ then by the adèlic form of Minkowski's second theorem (this is Theorem 3 of [2]) we have

$$
\left(\lambda_{1} \lambda_{2} \cdots \lambda_{M}\right)^{d} V(\mathscr{S}) \leq 2^{d M} .
$$

Using (2.3) this may be written as

$$
\left(\lambda_{1} \lambda_{2} \cdots \lambda_{M}\right) \leq c_{k}(M)^{M}\left(\prod_{v}\left|\xi_{v}\right|_{v}^{-M} H_{v}\left(A_{v}\right)\right) .
$$

To simplify the statement of the following lemma we write

$$
G(\mathbf{x})=\prod_{v} H_{v}\left\{\left(1_{N}-P_{v}\right) \mathbf{x}\right\}
$$

for $\mathrm{x} \in k$ and $P_{v}=P_{v}\left(\mathscr{A}_{v}\right)$.

Lemma 3. Let $\xi$ and $\zeta$ be linearly independent vectors in $(k)^{N} \backslash \mathscr{A}$. Then there exists a scalar $\alpha \neq 0$ in $k$ such that

$$
G(\alpha \boldsymbol{\zeta}+\boldsymbol{\xi}) \leq 2 c_{k}(1) \max \{G(\boldsymbol{\zeta}), G(\boldsymbol{\xi})\} .
$$

Proof. We use the inequality (2.4) with $M=1, A_{v}=\left(1_{N}-P_{v}\right) \zeta$, and $\xi_{v}=$ $\left(1_{N}-P_{v}\right) \xi$. For almost all finite places we have

$$
\left\|A_{v}\right\|_{v}=\left\|\boldsymbol{\xi}_{v}\right\|_{v}=1
$$

and therefore $S_{v}=\left(O_{v}\right)^{M}$ at almost all finite places. Hence the inequality (2.4) holds. It follows that there exists $\alpha \neq 0$ in $k$ such that $\alpha \in \lambda \mathscr{S}$ for all $\lambda>\lambda_{1}$. That is,

$$
\left\|\left(1_{N}-P_{v}\right) \alpha \zeta\right\|_{v} \leq \lambda_{1}\left\|\left(1_{N}-P_{v}\right) \xi\right\|_{v}
$$

if $v \mid \infty$ and

$$
\left\|\left(1_{N}-P_{v}\right) \alpha \xi\right\|_{v} \leq\left\|\left(1_{N}-P_{v}\right) \xi\right\|_{v}
$$


if $v+\infty$. Thus we have

$$
\prod_{v}\left|\left(1_{N}-P_{v}\right)(\alpha \boldsymbol{\zeta}+\boldsymbol{\xi})\right|_{v} \leq\left\{\prod_{v \mid \infty}\left(1+\lambda_{1}\right)^{d_{v} / d}\right\} \prod_{v}\left|\left(1_{N}-P_{v}\right) \boldsymbol{\xi}\right|_{v},
$$

or

$$
G(\alpha \boldsymbol{\zeta}+\boldsymbol{\xi}) \leq\left(1+\lambda_{1}\right) G(\boldsymbol{\xi}) .
$$

Of course (2.4) may be written as

$$
\lambda_{1} \leq c_{k}(1) G(\boldsymbol{\xi})^{-1} G(\boldsymbol{\zeta})
$$

and therefore (2.5) implies that

$$
G(\alpha \boldsymbol{\zeta}+\boldsymbol{\xi}) \leq G(\boldsymbol{\xi})+c_{k}(1) G(\boldsymbol{\zeta}) \leq 2 c_{k}(1) \max \{G(\boldsymbol{\zeta}), G(\boldsymbol{\xi})\} .
$$

This proves the lemma.

Let $F(\mathbf{x}, \mathbf{y})$ be the bilinear form defined in (1.1) by the $N \times N$ symmetric matrix $\Phi=\left(\varphi_{m n}\right)$. At each place $v$ of $k$ the local height $\mathscr{H}_{v}$ is defined by

$$
\begin{gathered}
\mathscr{H}_{v}(\Phi)=\max _{1 \leq m, n \leq N}\left|\varphi_{m n}\right|_{v} \quad \text { if } v \nmid \infty, \\
\mathscr{H}_{v}(\Phi)=\left\{\sum_{m=1}^{N} \sum_{n=1}^{N}\left\|\varphi_{m n}\right\|_{v}^{2}\right\}^{d_{v} / 2 d} \quad \text { if } v \mid \infty .
\end{gathered}
$$

The global height of $\Phi$ is then given by

$$
\mathscr{H}(\Phi)=\prod_{v} \mathscr{H}_{v}(\Phi)
$$

If $\boldsymbol{\xi}_{v}$ and $\boldsymbol{\zeta}_{v}$ are vectors in $\left(k_{v}\right)^{N}$ we have

$$
\left|F\left(\boldsymbol{\xi}_{v}, \boldsymbol{\zeta}_{v}\right)\right|_{v} \leq \mathscr{H}_{v}(\Phi)\left|\boldsymbol{\xi}_{v}\right|_{v}\left|\boldsymbol{\zeta}_{v}\right|_{v} .
$$

This follows immediately from the ultrametric inequality if $v+\infty$ and from the Cauchy-Schwarz inequality if $v \mid \infty$.

Now suppose that $\xi$ and $\zeta$ are vectors in $k^{N}, F$ vanishes identically on $\mathscr{A} \subseteq \mathscr{Z} \subseteq k^{N}$ and $\zeta \in \mathscr{A}^{\perp}$, where

$$
\mathscr{A}^{\perp}=\{\mathbf{z} \in \mathscr{Z}: F(\mathbf{a}, \mathbf{z})=0 \text { for all } \mathbf{a} \in \mathscr{A}\} .
$$

By continuity $F$ vanishes identically on the completion $\mathscr{A}_{v}$ of $\mathscr{A}$ in $\left(k_{v}\right)^{N}$. It follows that $F\left(P_{v} \xi, \zeta\right)=0$ and therefore

$$
F(\boldsymbol{\xi}, \zeta)=F\left(\left(1_{N}-P_{v}\right) \boldsymbol{\xi}, \zeta\right) .
$$


If $F(\xi, \zeta) \neq 0$, then $(2.6),(2.7)$ and the product formula imply that

$$
\begin{aligned}
1 & =\prod_{v}|F(\boldsymbol{\xi}, \zeta)|_{v} \\
& =\prod_{v}\left|F\left(\left(1_{N}-P_{v}\right) \boldsymbol{\xi}, \zeta\right)\right|_{v} \\
& \leq \prod_{v}\left\{\mathscr{C}_{v}(\Phi) H_{v}\left(\left(1_{N}-P_{v}\right) \xi\right) H_{v}(\zeta)\right\} \\
& =\mathscr{H}(\Phi)\left\{\prod_{v} H_{v}\left(\left(1_{N}-P_{v}\right) \xi\right)\right\} H(\zeta) .
\end{aligned}
$$

This type of argument will be used frequently.

\section{TWo Basic THEOREMS}

Our proof of Theorem 1 divides naturally into two parts and each part can be easily formulated as a separate result. We assume throughout $\S \S 3-6$ of this paper that the hypotheses in Theorem 1 hold. Then among all $L$-dimensional subspaces contained in $\mathscr{Z}^{(0)}$ let $\mathscr{X}$ be an $L$-dimensional subspace with minimal height. As the set of $L$-dimensional subspaces of $\mathscr{Z}$ having height less than a positive constant is a finite set, such a subspace $\mathscr{Z}$ clearly exists. We define

$$
\mathscr{X}^{\perp}=\{\mathbf{z} \in \mathscr{Z}: F(\mathbf{x}, \mathbf{z})=0 \text { for all } \mathbf{x} \in \mathscr{X}\} .
$$

Since $F$ restricted to $\mathscr{X}$ has rank $r>M-L$, it follows that $\mathscr{X}^{\perp}$ is a proper subspace of $\mathscr{Z}$.

Theorem 4. Suppose that $\xi$ is a vector in $\mathscr{Z} \backslash \mathscr{X}^{\perp}$ and let $\mathscr{Y}$ be the $(L+1)$ dimensional subspace

$$
\mathscr{Y}=\operatorname{span}_{k}\{\mathscr{X}, \xi\} \text {. }
$$

Then there exists a subspace $\mathscr{X}^{\prime} \subseteq \mathscr{Y}$ such that

(i) $F$ vanishes identically on $\mathscr{X}^{\prime}$,

(ii) $\operatorname{dim}\left(\mathscr{X}^{\prime}\right)=L$ and $\operatorname{dim}\left(\mathscr{Z} \cap \mathscr{X}^{\prime}\right)=L-1$,

(iii) $H(\mathscr{X})^{2} \leq H(\mathscr{Z}) H\left(\mathscr{X}^{\prime}\right) \leq 2 \mathscr{H}(\Phi) H(\mathscr{Y})^{2}$,

(iv) $1 \leq 2 \mathscr{H}(\Phi)\left\{\prod_{v} H_{v}\left(\left(1_{N}-P_{v}\right) \xi\right)\right\}^{2}$,

where $P_{v}=P_{v}\left(\mathscr{Z}_{v}\right)$ is projection onto the completion $\mathscr{X}_{v}$ of $\mathscr{X}$ in $\left(k_{v}\right)^{N}$.

The proof of this result is similar to our proof of [14, Theorem 1]. In fact we have made some technical simplifications which lead to the sharper inequality (1.6).

Theorem 5. There exist $M-L$ linearly independent vectors $\mathbf{z}_{1}, \mathbf{z}_{2}, \ldots, \mathbf{z}_{M-L}$ in $\mathscr{Z} \backslash \mathscr{Z}^{\perp}$ such that

$$
\mathscr{Z}=\operatorname{span}_{k}\left\{\mathscr{X}, \mathbf{z}_{1}, \mathbf{z}_{2}, \ldots, \mathbf{z}_{M-L}\right\},
$$

and each of the subspaces

$$
\mathscr{Y}_{l}=\operatorname{span}_{k}\left\{\mathscr{X}, \mathbf{z}_{l}\right\}, \quad l=1,2, \ldots, M-L,
$$


satisfies

$$
H\left(\mathscr{Y}_{l}\right) \leq 2 c_{k}(1)\left\{c_{k}(M-L)\right\}^{M-L}\{2 \mathscr{H}(\Phi)\}^{(M-L-1) / 2} H(\mathscr{Z}) .
$$

In order to prove Theorem 1 from these results we set $\mathscr{Z}=\mathscr{X}_{0}$. Then with $\mathbf{z}_{1}, \mathbf{z}_{2}, \ldots, \mathbf{z}_{M-L}$ as in Theorem 5 we apply Theorem 4 with $\xi=\mathbf{z}_{l}$. It follows that the subspace $\mathscr{Y}_{l}=\operatorname{span}\left\{\mathscr{X}_{0}, \mathbf{z}_{l}\right\}$ contains an $L$-dimensional subspace $\mathscr{X}_{l}=\mathscr{X}_{l}^{\prime}$ such that $\mathscr{X}_{l} \subseteq \mathscr{Z}^{(0)}, \operatorname{dim}\left(\mathscr{X}_{0} \cap \mathscr{X}_{l}\right)=L-1$, and

$$
\operatorname{span}\left\{\mathscr{X}_{0}, \mathscr{X}_{1}, \ldots, \mathscr{X}_{M-L}\right\}=\mathscr{Z} \text {. }
$$

Using (iii) of Theorem 4 we have

$$
H\left(\mathscr{X}_{0}\right)^{2} \leq H\left(\mathscr{X}_{0}\right) H\left(\mathscr{X}_{l}\right) \leq 2 \mathscr{H}(\Phi) H\left(\mathscr{Y}_{l}\right)^{2} .
$$

Then (3.3) and (3.4) combine to give exactly the estimate (1.2) in the statement of Theorem 1.

\section{Proof of Theorem 4}

Let $\left\{\mathbf{x}_{1}, \mathbf{x}_{2}, \ldots, \mathbf{x}_{L}\right\}$ be a basis for $\mathscr{X}$. Since $\xi \in \mathscr{Z} \backslash \mathscr{X}^{\perp}$ it follows that

$$
F\left(\mathbf{x}_{l}, \boldsymbol{\xi}\right) \neq 0 \text { for some } l, 1 \leq l \leq L \text {. }
$$

Obviously $\left\{\mathbf{x}_{1}, \ldots, \mathbf{x}_{L}, \boldsymbol{\xi}\right\}$ is a basis for $\mathscr{Y}$. Define $\mathscr{X}^{\prime} \subseteq \mathscr{Y}$ by

$$
\mathscr{X}^{\prime}=\left\{\sum_{l=1}^{L} \alpha_{l} \mathbf{x}_{l}+\beta \boldsymbol{\xi}: \alpha_{l} \in k, \beta \in k, \text { and } \sum_{l=1}^{L} \alpha_{l} F\left(\mathbf{x}_{l}, \boldsymbol{\xi}\right)+\frac{1}{2} \beta F(\boldsymbol{\xi})=0\right\} \text {. }
$$

In view of $(4.1), \mathscr{X}^{\prime}$ is a subspace of $\mathscr{Y}$ with

$$
\operatorname{dim}\left(\mathscr{X}^{\prime}\right)=L \quad \text { and } \quad \operatorname{dim}\left(\mathscr{X} \cap \mathscr{X}^{\prime}\right)=L-1 .
$$

In particular, $\mathscr{X}$ and $\mathscr{X}^{\prime}$ are distinct subspaces. If

$$
\mathbf{y}=\mathbf{x}+\beta \boldsymbol{\xi}, \quad \mathbf{x} \in \mathscr{X}, \beta \in k,
$$

is a vector in $\mathscr{Y}$ then

$$
\begin{aligned}
F(\mathbf{y}) & =2 F(\mathbf{x}, \beta \boldsymbol{\xi})+F(\beta \boldsymbol{\xi}) \\
& =2 \beta F\left(\mathbf{x}+\frac{1}{2} \beta \boldsymbol{\xi}, \boldsymbol{\xi}\right) .
\end{aligned}
$$

This shows that $\beta=0$ is equivalent to $\mathbf{y} \in \mathscr{X}$ and $F\left(\mathbf{x}+\frac{1}{2} \beta \boldsymbol{\xi}, \boldsymbol{\xi}\right)=0$ is equivalent to $y \in \mathscr{X}^{\prime}$. Therefore (i) and (ii) of Theorem 4 hold for the subspace $\mathscr{X}^{\prime}$.

At each place $v$ of $k$ let $\mathscr{X}_{v} \subseteq\left(k_{v}\right)^{N}$ be the completion of $\mathscr{Z}$ and let $\mathscr{X}_{v}^{\prime} \subseteq\left(k_{v}\right)^{N}$ be the completion of $\mathscr{X}^{\prime}$. Then set

$$
P_{v}=P_{v}\left(\mathscr{Q}_{v}\right), \quad P_{v}^{\prime}=P_{v}\left(\mathscr{X}_{v}^{\prime}\right),
$$

and

$$
Q_{v}=\frac{1}{2}\left(1_{N}-P_{v}\right)
$$


By continuity $F$ must vanish identically on $\mathscr{X}_{v} \cup \mathscr{X}_{v}^{\prime}$. With y given by (4.2) we have

$$
\begin{aligned}
F\left(\mathbf{x}+\frac{1}{2} \beta \boldsymbol{\xi}, \boldsymbol{\xi}\right) & =F(\mathbf{x}, \boldsymbol{\xi})+\frac{1}{2} \beta F(\boldsymbol{\xi}) \\
& =F\left(\mathbf{x},\left(1_{N}-P_{v}\right) \boldsymbol{\xi}\right)+\frac{1}{2} \beta F\left(\left(1_{N}+P_{v}\right) \boldsymbol{\xi},\left(1_{N}-P_{v}\right) \xi\right) \\
& =F\left(Q_{v} \mathbf{x},\left(1_{N}-P_{v}\right) \boldsymbol{\xi}\right)+F\left(\beta Q_{v} \boldsymbol{\xi},\left(1_{N}-P_{v}\right) \boldsymbol{\xi}\right) \\
& =F\left(Q_{v} \mathbf{y},\left(1_{N}-P_{v}\right) \boldsymbol{\xi}\right) .
\end{aligned}
$$

If we combine (4.3) and (4.4) we find that

$$
F(\mathbf{y})=2 \beta F\left(Q_{v} \mathbf{y},\left(1_{N}-P_{v}\right) \xi\right)
$$

at each place $v$ of $k$. It follows that $\mathrm{y} \in \mathscr{X}^{\prime}$ if and only if

$$
F\left(Q_{v} \mathbf{y},\left(1_{N}-P_{v}\right) \xi\right)=0 .
$$

Now select $\mathbf{y} \in \mathscr{Y} \backslash\left(\mathscr{X} \cup \mathscr{X}^{\prime}\right)$. Then $P_{v}^{\prime} \mathbf{y} \in \mathscr{X}_{v}^{\prime}$ and so at each place $v$

$$
F\left(Q_{v} P_{v}^{\prime} \mathbf{y},\left(1_{N}-P_{v}\right) \xi\right)=0 .
$$

Combining (4.5) and (4.6) we obtain

$$
0 \neq F(\mathbf{y})=2 \beta F\left(Q_{v}\left(1_{N}-P_{v}^{\prime}\right) \mathbf{y},\left(1_{N}-P_{v}\right) \xi\right) \text {. }
$$

Next we apply the product formula to (4.7) and use the basic inequality (2.6) at each place. This leads to

$$
\begin{aligned}
1 & =\prod_{v}\left|(2 \beta)^{-1} F(\mathbf{y})\right|_{v} \\
& \leq \mathscr{H}(\Phi)\left\{\prod_{v} H_{v}\left(Q_{v}\left(1_{N}-P_{v}^{\prime}\right) \mathbf{y}\right)\right\}\left\{\prod_{v} H_{v}\left(\left(1_{N}-P_{v}\right) \xi\right)\right\} .
\end{aligned}
$$

By [14, Lemma 8] we may remove the operator $Q_{v}$ from the right-hand side of (4.8) while compensating with an extra factor of 2 . In this way we find that

$$
1 \leq 2 \mathscr{H}(\Phi)\left\{\prod_{v} H_{v}\left(\left(1_{N}-P_{v}^{\prime}\right) \mathbf{y}\right)\right\}\left\{\prod_{v} H_{v}\left(\left(1_{N}-P_{v}\right) \xi\right)\right\} .
$$

Finally we multiply both sides of $(4.9)$ by $H(\mathscr{X}) H\left(\mathscr{X}^{\prime}\right)$ and apply (2.1). This establishes the inequality

$$
\begin{aligned}
H(\mathscr{X}) H\left(\mathscr{X}^{\prime}\right) & \leq 2 \mathscr{H}(\Phi) H\left(\operatorname{span}_{k}\left\{\mathscr{X}^{\prime}, \mathbf{y}\right\}\right) H\left(\operatorname{span}_{k}\{\mathscr{X}, \xi\}\right) \\
& =2 \mathscr{H}(\Phi) H(\mathscr{Y})^{2},
\end{aligned}
$$

which is exactly the second inequality in (iii) of the theorem.

Now $\mathscr{X} \subseteq \mathscr{Z}^{(0)}$ has minimal height among all $L$-dimensional subspaces contained in $\mathscr{Z}^{(0)}$. Therefore (4.10) immediately implies that

$$
\begin{aligned}
H(\mathscr{X})^{2} & \leq H(\mathscr{X}) H\left(\mathscr{X}^{\prime}\right) \leq 2 \mathscr{H}(\Phi) H(\mathscr{Y})^{2} \\
& =2 \mathscr{H}(\Phi) H(\mathscr{X})^{2}\left\{\prod_{v} H_{v}\left(\left(1_{N}-P_{v}\right) \xi\right)\right\}^{2} .
\end{aligned}
$$


Of course (4.11) is (iv) and the first inequality in (iii) of the theorem.

\section{A NESTED SEQUENCE OF SUBSPACES}

In this section we identify several objects connected with $F, \mathscr{X}$ and $\mathscr{Z}$ which will be used in our proof of Theorem 5. Again we suppose that $\left\{\mathbf{x}_{1}, \mathbf{x}_{2}\right.$, $\left.\ldots, \mathbf{x}_{L}\right\}$ is a basis for $\mathscr{X}$ and we write $P_{v}=P_{v}\left(\mathscr{X}_{v}\right)$ for the projection operator which maps $\left(k_{v}\right)^{N}$ onto the completion $\mathscr{X}_{v}$ of $\mathscr{X}$ at each place $v$. To simplify some expressions we write

$$
G(\mathbf{z})=\prod_{v} H_{v}\left(\left(1_{N}-P_{v}\right) \mathbf{z}\right)
$$

if $\mathbf{z} \in \mathscr{Z}$. By [14, Theorem 10] there exists a basis $\left\{\mathbf{x}_{1}, \mathbf{x}_{2}, \ldots, \mathbf{x}_{L}, \mathbf{y}_{1}, \mathbf{y}_{2}, \ldots\right.$, $\left.\mathbf{y}_{M-L}\right\}$ of $\mathscr{Z}$ such that

$$
\prod_{l=1}^{M-L} G\left(\mathbf{y}_{l}\right) \leq\left\{c_{k}(M-L)\right\}^{M-L} H(\mathscr{Z}) H(\mathscr{X})^{-1} .
$$

Also, by reordering if necessary, we may assume that the numbers $G\left(\mathbf{y}_{l}\right)$ are increasing for $l=1,2, \ldots, M-L$. We use the vectors in $\left\{\mathbf{y}_{1}, \mathbf{y}_{2}, \ldots, \mathbf{y}_{M-L}\right\}$ to define a nested sequence of subspaces

$$
\mathscr{A}_{l}=\operatorname{span}_{k}\left\{\mathbf{x}_{1}, \mathbf{x}_{2}, \ldots, \mathbf{x}_{L}, \mathbf{y}_{1}, \mathbf{y}_{2}, \ldots, \mathbf{y}_{l}\right\},
$$

for $l=0,1,2, \ldots, M-L$. Thus we have

$$
\mathscr{X}=\mathscr{A}_{0} \subseteq \mathscr{A}_{1} \subseteq \mathscr{A}_{2} \subseteq \cdots \subseteq \mathscr{A}_{M-L}=\mathscr{Z} \text {. }
$$

Obviously $\operatorname{dim}\left(\mathscr{A}_{l}\right)=L+l$. For each choice of $l=0,1,2, \ldots, M-L$ we set

$$
\mathscr{A}_{l}^{\perp}=\left\{\mathbf{z} \in \mathscr{Z}: F(\mathbf{a}, \mathbf{z})=0 \text { for all } \mathbf{a} \in \mathscr{A}_{l}\right\} .
$$

Clearly each $\mathscr{A}_{l}^{\perp}$ is a subspace of $\mathscr{Z}$ and

$$
\mathscr{Z}^{\perp}=\mathscr{A}_{M-L}^{\perp} \subseteq \mathscr{A}_{M-L-1}^{\perp} \subseteq \cdots \subseteq \mathscr{A}_{1}^{\perp} \subseteq \mathscr{A}_{0}^{\perp}=\mathscr{X}^{\perp} \subseteq \mathscr{Z} .
$$

Concerning the dimension of $\mathscr{A}_{l}^{\perp}$ we have

$$
\operatorname{dim}\left(\mathscr{A}_{l}\right)+\operatorname{dim}\left(\mathscr{A}_{l}^{\perp}\right)=\operatorname{dim}(\mathscr{Z})+\operatorname{dim}\left(\mathscr{A}_{l} \cap \mathscr{Z}^{\perp}\right) .
$$

Since $r>M-L$ it follows that

$$
\begin{aligned}
\operatorname{dim}\left(\mathscr{A}_{l}^{\perp}\right) & \leq \operatorname{dim}(\mathscr{Z})+\operatorname{dim}\left(\mathscr{Z}^{\perp}\right)-\operatorname{dim}\left(\mathscr{A}_{l}\right) \\
& =M+(M-r)-(L+l) \\
& \leq M-l-1 .
\end{aligned}
$$

The quadratic form $F$ vanishes identically on $\mathscr{X}=\mathscr{A}_{0}$ but does not vanish identically on $\mathscr{Z}=\mathscr{A}_{M-L}$. Hence there exists a unique integer $s, 0 \leq s \leq M-$ $L-1$, such that $F$ vanishes identically on $\mathscr{A}_{s}$ but $F$ does not vanish identically on $\mathscr{A}_{s+1}$. For the subspace $\mathscr{A}_{s}$ we must have $\mathscr{A}_{s} \subseteq \mathscr{A}_{s}^{\perp}$, and therefore by (5.2)

$$
L+s=\operatorname{dim}\left(\mathscr{A}_{s}\right) \leq \operatorname{dim}\left(\mathscr{A}_{s}^{\perp}\right) \leq M-s-1 .
$$

This shows that $0 \leq s \leq \frac{1}{2}(M-L-1)$. 
Lemma 6. If $\mathbf{y}_{s+1} \in \mathscr{A}_{s}^{\perp}$ then

$$
1 \leq \mathscr{H}(\Phi) G\left(\mathbf{y}_{s+1}\right)^{2}
$$

Proof. By the definition of $s, F$ does not vanish identically on

$$
\mathscr{A}_{s+1}=\operatorname{span}_{k}\left\{\mathscr{A}_{s}, \mathbf{y}_{s+1}\right\} \text {. }
$$

But

$$
F(\mathbf{a})=0 \text { and } F\left(\mathbf{a}, \mathbf{y}_{s+1}\right)=0
$$

for all $\mathbf{a} \in \mathscr{A}_{s}$. It follows that $F\left(\mathbf{y}_{s+1}\right) \neq 0$. Also, (5.3) implies that at each place $v$

$$
F\left(P_{v} \mathbf{y}_{s+1}\right)=0 \text { and } F\left(P_{v} \mathbf{y}_{s+1}, \mathbf{y}_{s+1}\right)=0
$$

Therefore, we have

$$
\begin{aligned}
F\left(\mathbf{y}_{s+1}\right) & =F\left(\left(1_{N}-P_{v}\right) \mathbf{y}_{s+1}, \mathbf{y}_{s+1}\right) \\
& =F\left(\left(1_{N}-P_{v}\right) \mathbf{y}_{s+1}\right) .
\end{aligned}
$$

We apply (2.6) and the product formula to conclude that

$$
\begin{aligned}
1 & =\prod_{v}\left|F\left(\mathbf{y}_{s+1}\right)\right|_{v} \\
& =\prod_{v}\left|F\left(\left(1_{N}-P_{v}\right) \mathbf{y}_{s+1}\right)\right|_{v} \\
& \leq \mathscr{H}(\Phi) G\left(\mathbf{y}_{s+1}\right)^{2} .
\end{aligned}
$$

Lemma 7. Suppose that $1 \leq s$ and $\xi$ is a vector in $\mathscr{Z} \backslash \mathscr{A}_{s}^{\perp}$. Let $m$ be the smallest integer in the set $\{1,2, \ldots, s\}$ such that $\xi$ is contained in $\mathscr{Z} \backslash \mathscr{A}_{m}^{\perp}$. Then we have

$$
1 \leq \mathscr{H}(\Phi) G\left(\mathbf{y}_{m}\right) G(\boldsymbol{\xi})
$$

Proof. First we assume that $m=1$. Then $\xi \in \mathscr{Z} \backslash \mathscr{A}_{1}^{\perp}$ and therefore the linear form $\mathbf{a} \rightarrow F(\mathbf{a}, \xi)$ is not trivial on $\mathscr{A}_{1}$. It follows that

$$
\mathscr{B}_{0}=\left\{\mathbf{a} \in \mathscr{A}_{1}: F(\mathbf{a}, \boldsymbol{\xi})=0\right\}
$$

is an $L$-dimensional subspace of $\mathscr{A}_{1}$. As $1 \leq s, F$ vanishes identically on $\mathscr{A}_{1}$ and also on $\mathscr{B}_{0}$. Since $\mathscr{X}=\mathscr{A}_{0}$ has minimal height among the $L$-dimensional subspaces in $\mathscr{Z}^{(0)}$, we have

$$
H\left(\mathscr{A}_{0}\right) \leq H\left(\mathscr{B}_{0}\right) \text {. }
$$
have

Now let $\zeta \in \mathscr{A}_{1} \backslash \mathscr{B}_{0}$ so that $F(\zeta, \xi) \neq 0$. From the definition of $\mathscr{B}_{0}$ we

$$
F\left(P_{v}\left(\mathscr{B}_{0}\right) \zeta, \xi\right)=0
$$

at each place $v$. Since $F$ vanishes identically on $\mathscr{A}_{1}$ we also have

$$
F\left(\left(1_{N}-P_{v}\left(\mathscr{B}_{0}\right)\right) \zeta, P_{v}\left(\mathscr{A}_{0}\right) \xi\right)=0 .
$$


Using (5.6) and (5.7) we obtain the identity

$$
\begin{aligned}
F(\boldsymbol{\zeta}, \boldsymbol{\xi}) & =F\left(\left(1_{N}-P_{v}\left(\mathscr{B}_{0}\right)\right) \boldsymbol{\zeta}, \boldsymbol{\xi}\right) \\
& =F\left(\left(1_{N}-P_{v}\left(\mathscr{B}_{0}\right)\right) \boldsymbol{\zeta},\left(1_{N}-P_{v}\left(\mathscr{A}_{0}\right)\right) \boldsymbol{\xi}\right)
\end{aligned}
$$

at each place $v$. Again we apply (2.6) and the product formula to conclude that

$$
\begin{aligned}
1 & =\prod_{v}|F(\boldsymbol{\zeta}, \boldsymbol{\xi})|_{v} \\
& \leq \mathscr{H}(\boldsymbol{\Phi})\left\{\prod_{v} H_{v}\left(\left(1_{N}-P_{v}\left(\mathscr{B}_{0}\right)\right) \boldsymbol{\zeta}\right)\right\}\left\{\prod_{v} H_{v}\left(\left(1_{N}-P_{v}\left(\mathscr{A}_{0}\right)\right) \boldsymbol{\xi}\right)\right\} .
\end{aligned}
$$

Next we multiply both sides of $(5.8)$ by $H\left(\mathscr{B}_{0}\right)$ and use $(2.1)$ and (5.5). In this way we obtain

$$
\begin{aligned}
H\left(\mathscr{A}_{0}\right) & \leq H\left(\mathscr{B}_{0}\right) \leq \mathscr{H}(\Phi) H\left(\mathscr{A}_{1}\right)\left\{\prod_{v} H_{v}\left(\left(1_{N}-P_{v}\left(\mathscr{A}_{0}\right)\right) \boldsymbol{\xi}\right)\right\} \\
& =\mathscr{H}(\boldsymbol{\Phi}) H\left(\mathscr{A}_{0}\right)\left\{\prod_{v} H_{v}\left(\left(1_{N}-P_{v}\left(\mathscr{A}_{0}\right)\right) \mathbf{y}_{1}\right)\right\}\left\{\prod_{v} H_{v}\left(\left(1_{N}-P_{v}\left(\mathscr{A}_{0}\right)\right) \boldsymbol{\xi}\right)\right\},
\end{aligned}
$$

which is (5.4) when $m=1$.

To complete the proof we consider the case $2 \leq m \leq s$. Then $\xi \in \mathscr{Z} \backslash \mathscr{A}_{m}^{\perp}$ and $\boldsymbol{\xi} \in \mathscr{A}_{m-1}^{\perp}$. Again the linear form $\mathbf{a} \rightarrow F(\mathbf{a}, \boldsymbol{\xi})$ is not trivial on $\mathscr{A}_{m}$ so that

$$
\left\{\mathbf{a} \in \mathscr{A}_{m}: F(\mathbf{a}, \boldsymbol{\xi})=0\right\}
$$

is an $(L+m-1)$-dimensional subspace of $\mathscr{A}_{m}$. On the other hand, $\mathscr{A}_{m-1} \subseteq \mathscr{A}_{m}$, $\boldsymbol{\xi} \in \mathscr{A}_{m-1}^{\perp}$ and therefore $\mathbf{a} \rightarrow F(\mathbf{a}, \boldsymbol{\xi})$ is identically zero on $\mathscr{A}_{m-1}$. In other words,

$$
\mathscr{A}_{m-1}=\left\{\mathbf{a} \in \mathscr{A}_{m}: F(\mathbf{a}, \boldsymbol{\xi})=0\right\} .
$$

The vector $\mathbf{y}_{m}$ is in $\mathscr{A}_{m} \backslash \mathscr{A}_{m-1}$ and therefore $F\left(\mathbf{y}_{m}, \boldsymbol{\xi}\right) \neq 0$. As in the first part of the proof we have

$$
\begin{aligned}
F\left(\mathbf{y}_{m}, \boldsymbol{\xi}\right) & =F\left(\left(1_{N}-P_{v}\left(\mathscr{A}_{0}\right)\right) \mathbf{y}_{m}, \boldsymbol{\xi}\right) \\
& =F\left(\left(1_{N}-P_{v}\left(\mathscr{A}_{0}\right)\right) \mathbf{y}_{m},\left(1_{N}-P_{v}\left(\mathscr{A}_{0}\right)\right) \boldsymbol{\xi}\right) .
\end{aligned}
$$

Using (2.1), (5.9) and the product formula we find that

$$
1=\prod_{v}\left|F\left(\mathbf{y}_{m}, \boldsymbol{\xi}\right)\right|_{v} \leq \mathscr{H}(\Phi) G\left(\mathbf{y}_{m}\right) G(\boldsymbol{\xi})
$$

This proves the lemma.

Lemma 8. The vectors $\mathbf{y}_{1}, \mathbf{y}_{2}, \ldots, \mathbf{y}_{M-L-1}$ satisfy

$$
1 \leq\{2 \mathscr{H}(\Phi)\}^{(M-L-1) / 2} \prod_{l=1}^{M-L-1} G\left(\mathbf{y}_{l}\right)
$$


The vector $\mathbf{y}_{M-L}$ satisfies

$$
G\left(\mathbf{y}_{M-L}\right) \leq\left\{c_{k}(M-L)\right\}^{M-L}\{2 \mathscr{H}(\Phi)\}^{(M-L-1) / 2} H(\mathscr{Z}) H(\mathscr{X})^{-1} .
$$

Proof. The inequalities (5.1) and (5.10) clearly imply that (5.11) holds. Therefore it suffices to establish only (5.10). First we consider the case $s=0$. If $\mathbf{y}_{1} \in \mathscr{A}_{0}^{\perp}$ then

$$
1 \leq \mathscr{H}(\Phi) G\left(\mathbf{y}_{1}\right)^{2}
$$

holds by Lemma 6. If $\mathbf{y}_{1} \in \mathscr{Z} \backslash \mathscr{A}_{0}^{\perp}$ we may appeal to Theorem 4 part (iv) with $\boldsymbol{\xi}=\mathbf{y}_{1}$. We find that

$$
1 \leq 2 \mathscr{H}(\Phi) G\left(\mathbf{y}_{1}\right)^{2} .
$$

Thus (5.12) holds generally if $s=0$. Since the numbers $G\left(\mathbf{y}_{l}\right)$ are increasing for $l=1,2, \ldots, M-L$, the desired inequality (5.10) follows immediately.

For the remainder of the proof we assume that $1 \leq s$. Let $0 \leq l \leq s$ so that

$$
\mathscr{A}_{l} \subseteq \mathscr{A}_{s} \subseteq \mathscr{A}_{s}^{\perp} \subseteq \mathscr{A}_{l}^{\perp} \text {. }
$$

In particular,

$$
\mathscr{A}_{s}=\operatorname{span}_{k}\left\{\mathscr{A}_{0}, \mathbf{y}_{1}, \ldots, \mathbf{y}_{s}\right\} \subseteq \mathscr{A}_{l}^{\perp}
$$

and $\operatorname{dim}\left(\mathscr{A}_{l}^{\perp}\right) \leq M-l-1$ by (5.2). It follows that at most $M-L-s-l-1$ of the vectors in the set $\left\{\mathbf{y}_{s+1}, \mathbf{y}_{s+2}, \ldots, \mathbf{y}_{M-L}\right\}$ are also in $\mathscr{A}_{l}^{\perp}$. Alternatively, at least $l+1$ vectors in the set $\left\{\mathbf{y}_{s+1}, \mathbf{y}_{s+2}, \ldots, \mathbf{y}_{M-L}\right\}$ are also in $\mathscr{Z} \backslash \mathscr{A}_{l}^{\perp}$. Hence we may select distinct integers $i_{0}, i_{1}, \ldots, i_{s}$ in $\{s+1, s+2, \ldots, M-L\}$ so that

$$
\left\{\mathbf{y}_{i_{0}}, \mathbf{y}_{i_{1}}, \ldots, \mathbf{y}_{i_{l}}\right\} \subseteq \mathscr{Z} \backslash \mathscr{A}_{l}^{\perp}
$$

for each $l=0,1,2, \ldots, s$. By removing $\mathbf{y}_{M-L}$ if necessary, we may select distinct integers $j_{1}, j_{2}, \ldots, j_{s}$ in the set $\{s+1, s+2, \ldots, M-L-1\}$ so that

$$
\left\{\mathbf{y}_{j_{1}}, \mathbf{y}_{j_{2}}, \ldots, \mathbf{y}_{j_{l}}\right\} \subseteq \mathscr{Z} \backslash \mathscr{A}_{l}^{\perp}
$$

for $l=1,2, \ldots, s$. Since $\mathscr{Z} \backslash \mathscr{A}_{l}^{\perp} \subseteq \mathscr{Z} \backslash \mathscr{A}_{s}^{\perp}$ the hypotheses of Lemma 7 hold with $\boldsymbol{\xi}=\mathbf{y}_{j_{l}}$. The corresponding value of $m$ plainly satisfies $1 \leq m \leq l$. Thus by Lemma 7 we have

$$
1 \leq \mathscr{H}(\Phi) G\left(\mathbf{y}_{m}\right) G\left(\mathbf{y}_{j_{l}}\right) \leq \mathscr{H}(\Phi) G\left(\mathbf{y}_{l}\right) G\left(\mathbf{y}_{j_{l}}\right),
$$

for each $l=1,2, \ldots, s$.

Next we claim that

$$
1 \leq \mathscr{H}(\Phi) G\left(\mathbf{y}_{s+1}\right)^{2} .
$$

If $\mathbf{y}_{s+1} \in \mathscr{A}_{s}^{\perp}$ then this follows from Lemma 6. If $\mathbf{y}_{s+1} \in \mathscr{Z} \backslash \mathscr{A}_{s}^{\perp}$ we may apply Lemma 7 with $\boldsymbol{\xi}=\mathbf{y}_{s+1}$. Then we use the trivial inequality $G\left(\mathbf{y}_{m}\right) \leq G\left(\mathbf{y}_{s+1}\right)$ (where $1 \leq m \leq s$ ) to deduce that (5.14) holds in this case as well. 
Now suppose that $t$ is an integer, $s+1 \leq t \leq M-L-1$, but $t$ is not in the set $\left\{j_{1}, j_{2}, \ldots, j_{s}\right\}$. Obviously $G\left(\mathbf{y}_{s+1}\right) \leq G\left(\mathbf{y}_{t}\right)$ and therefore (5.14) implies that

$$
1 \leq \mathscr{H}(\Phi) G\left(\mathbf{y}_{t}\right)^{2}
$$

There are $M-L-2 s-1$ such integers $t$ so that (5.15) leads to the inequality

$$
1 \leq \mathscr{H}(\Phi)^{(M-L-1) / 2-s} \prod_{t} G\left(\mathbf{y}_{t}\right) .
$$

From (5.13) we have

$$
1 \leq \mathscr{H}(\Phi)^{s}\left\{\prod_{l=1}^{s} G\left(\mathbf{y}_{l}\right) G\left(\mathbf{y}_{j_{l}}\right)\right\} .
$$

Finally, the inequalities (5.16) and (5.17) combine to establish (5.10).

\section{Proof of Theorem 5}

We have already seen that there exists an integer $i_{0}, s+1 \leq i_{0} \leq M-L$, such that $\mathbf{y}_{i_{0}}$ is contained in $\mathscr{Z} \backslash \mathscr{A}_{0}^{\perp}=\mathscr{Z} \backslash \mathscr{X}^{\perp}$. Using $\mathbf{y}_{i_{0}}$ and the linearly independent vectors $\left\{\mathbf{y}_{1}, \mathbf{y}_{2}, \ldots, \mathbf{y}_{M-L}\right\}$ we define a second set of linearly independent vectors $\left\{\mathbf{z}_{1}, \mathbf{z}_{2}, \ldots, \mathbf{z}_{M-L}\right\}$ as follows.

(i) If $\mathbf{y}_{l} \in \mathscr{Z} \backslash \mathscr{X}^{\perp}$ we set $\mathbf{z}_{l}=\mathbf{y}_{l}$.

(ii) If $\mathbf{y}_{l} \in \mathscr{X}^{\perp}$ we select a scalar $\alpha_{l} \neq 0$ in $k$ such that

$$
G\left(\alpha_{l} \mathbf{y}_{l}+\mathbf{y}_{i_{0}}\right) \leq 2 c_{k}(1) \max \left\{G\left(\mathbf{y}_{l}\right), G\left(\mathbf{y}_{i_{0}}\right)\right\} .
$$

That such a scalar $\alpha_{l} \neq 0$ exists follows from Lemma 3. Then we set $\mathbf{z}_{l}=\alpha_{l} \mathbf{y}_{l}+\mathbf{y}_{i_{0}}$.

It is clear that

$$
\operatorname{span}_{k}\left\{\mathbf{y}_{1}, \mathbf{y}_{2}, \ldots, \mathbf{y}_{M-L}\right\}=\underset{k}{\operatorname{span}}\left\{\mathbf{z}_{1}, \mathbf{z}_{2}, \ldots, \mathbf{z}_{M-L}\right\}
$$

and therefore (3.1) holds. Also, each vector $\mathbf{z}_{l}$ is in $\mathscr{Z} \backslash \mathscr{X}^{\perp}$.

To complete the proof we note that

$$
G\left(\mathbf{z}_{l}\right) \leq 2 c_{k}(1) G\left(\mathbf{y}_{M-L}\right)
$$

for each $l, 1 \leq l \leq M-L$. If $\mathscr{Y}_{l}$ is defined by (3.2) then

$$
H\left(\mathscr{Y}_{l}\right)=H(\mathscr{X}) G\left(\mathbf{z}_{l}\right)
$$

The inequality (3.3) plainly follows from (5.11), (6.1) and (6.2).

\section{Proof of Theorem 2}

Since the rank of $F$ restricted to $\mathscr{Z}$ is $r$ there exists a subspace $\mathscr{Y} \subseteq \mathscr{Z}$ such that $\mathscr{Y}$ has dimension $r$ and $F$ restricted to $\mathscr{Y}$ is nonsingular. By Theorem 10 of [14] there exists a basis $\left\{\mathbf{y}_{1}, \mathbf{y}_{2}, \ldots, \mathbf{y}_{r}\right\}$ of $\mathscr{Y}$ for which

$$
\prod_{l=1}^{r}\left\{\prod_{v} H_{v}\left\{\left(1_{N}-P_{v}\left(\mathscr{Z}^{\perp}\right)\right) \mathbf{y}_{l}\right\}\right\} \leq c_{k}(r)^{r} H(\mathscr{Z}) H\left(\mathscr{Z}^{\perp}\right)^{-1} \text {. }
$$


Let

$$
Y=\left(\mathbf{y}_{1} \mathbf{y}_{2} \cdots \mathbf{y}_{r}\right)
$$

denote the $N \times r$ matrix having $\mathbf{y}_{l}$ as its $l$ th column, $1 \leq l \leq r$. We must have

$$
\operatorname{det}\left\{Y^{T} \Phi Y\right\} \neq 0 \text {, }
$$

and therefore when we expand the determinant

$$
\sum_{\sigma} \operatorname{sgn}(\sigma) \prod_{l=1}^{r} F\left(\mathbf{y}_{l}, \mathbf{y}_{\sigma(l)}\right) \neq 0 .
$$

In (7.2) the sum is over all permutations $\sigma$ of the set $\{1,2, \ldots, r\}$. It follows that there exists a permutation $\tau$ of the set $\{1,2, \ldots, r\}$ such that

$$
\prod_{l=1}^{r} F\left(\mathbf{y}_{l}, \mathbf{y}_{\tau(l)}\right) \neq 0 \text {. }
$$

Using (2.1) and the product formula we find that

$$
\begin{aligned}
1 & =\prod_{l=1}^{r}\left\{\prod_{v}\left|F\left(\mathbf{y}_{l}, \mathbf{y}_{\tau(l)}\right)\right|_{v}\right\} \\
& =\prod_{l=1}^{r}\left\{\prod_{v}\left|F\left(\left(1_{N}-P_{v}\left(\mathscr{Z}^{\perp}\right)\right) \mathbf{y}_{l},\left(1_{N}-P_{v}\left(\mathscr{Z}^{\perp}\right)\right) \mathbf{y}_{\tau(l)}\right)\right|_{v}\right\} \\
& \leq \prod_{l=1}^{r}\left\{\mathscr{H}(\Phi) G\left(\mathbf{y}_{l}\right) G\left(\mathbf{y}_{\tau(l)}\right)\right\} \\
& =\mathscr{H}(\Phi)^{r} \prod_{l=1}^{r} G\left(\mathbf{y}_{l}\right)^{2}
\end{aligned}
$$

where we have written

$$
G(\mathbf{x})=\prod_{v} H_{v}\left\{\left(1_{N}-P_{v}\left(\mathscr{Z}^{\perp}\right)\right) \mathbf{x}\right\}
$$

Finally, from (7.1) and (7.3) we obtain the inequality

$$
1 \leq \mathscr{H}(\Phi)^{r}\left\{c_{k}(r)^{r} H(\mathscr{Z}) H\left(\mathscr{Z}^{\perp}\right)^{-1}\right\}^{2},
$$

which is the statement of the theorem.

\section{REFERENCES}

1. B. J. Birch and H. Davenport, Quadratic equations in several variables, Proc. Cambridge Philos. Soc. 54 (1958), 135-138.

2. E. Bombieri and J. Vaaler, On Siegel's lemma, Invent. Math. 73 (1983), 11-32.

3. J. W. S. Cassels, Bounds for the least solution of homogeneous quadratic equations, Proc. Cambridge Philos. Soc. 51 (1955), 262-264.

4. __ Addendum to the paper. Bounds for the least solution of homogeneous quadratic equations, Proc. Cambridge Philos. Soc. 52 (1956), 604. 
5. J. H. H. Chalk, Linearly independent zeros of quadratic forms over number fields, Monatsh. Math. 90 (1980), 13-25.

6. H. Davenport, Note on a theorem of Cassels, Proc. Cambridge Philos. Soc. 53 (1957), 539-540.

7. __, Homogeneous quadratic equations, Mathematika 18 (1971), 1-4.

8. S. Raghaven, Bounds of minimal solutions of diophantine equations, Nachr. Akad. Wiss. Gottingen Math. Phys. Kl. 9 (1975), 109-114.

9. H. P. Schlickewei, Kleine Nullstellen homogener quadratischer Gleichungen, Monatsh. Math. 100 (1985), 35-45.

10. H. P. Schlickewei and W. M. Schmidt, Quadartic geometry of numbers, Trans. Amer. Math. Soc. 301 (1987), 679-690.

11. W. M. Schmidt, On heights of algebraic subspaces and diophantine approximations, Ann. of Math. 85 (1967), 430-472.

12. __ Small zeros of quadratic forms, Trans. Amer. Math. Soc. 291 (1985), 87-102.

13. R. Schulze-Pillot, Small linearly independent zeros of quadratic forms, Monatsh. Math. 95 (1983), 241-249.

14. J. D. Vaaler, Small zeros of quadratic forms over number fields, Trans. Amer. Math. Soc. $\mathbf{3 0 2}$ (1987), 281-296.

15. G. L. Watson, Least solution of homogeneous quadratic equations, Proc. Cambridge Philos. Soc. 53 (1956), 541-543.

Department of Mathematics, The University of Texas at Austin, Austin, TeXas 78712 